\title{
Numerical modelling of plasticity induced by transcranial magnetic stimulation
}

\author{
M. T. Wilson - D. P. Goodwin . \\ P. W. Brownjohn · J. Shemmell · \\ J. N. J. Reynolds
}

Received: date / Accepted: date

\begin{abstract}
We use neural field theory and spike-timing dependent plasticity to make a simple but biophysically reasonable model of long-term plasticity changes in the cortex due to transcranial magnetic stimulation (TMS). We show how common TMS protocols can be captured and studied within existing neural field theory. Specifically, we look at repetitive TMS protocols such as theta burst stimulation and paired-pulse protocols. Continuous repetitive protocols result mostly in depression, but intermittent repetitive protocols in potentiation. A paired pulse protocol results in depression at short $(<\sim 10 \mathrm{~ms})$ and long $(>\sim 100 \mathrm{~ms})$ interstimulus intervals, but potentiation for mid-range intervals. The model is sensitive to the choice of neural populations that are driven by the TMS pulses, and to the parameters that describe plasticity, which may aid interpretation of the high variability in existing experimental results. Driving excitatory populations results in greater plasticity changes than driving inhibitory populations. Modelling also shows the merit in optimizing a TMS protocol based on an individual's electroencephalogram. Moreover, the model can be used to make predictions about protocols that may lead to improvements in repetitive TMS outcomes.
\end{abstract}

Keywords Transcranial Magnetic Stimulation · Plasticity · Modelling · Theta Burst Stimulation · Neural Field Theory

PACS 87.19.lw $\cdot 87.19 .1 \mathrm{l} \cdot 02.70 . \mathrm{Hm}$

M. T. Wilson · D. P. Goodwin

School of Engineering, Faculty of Science and Engineering, University of Waikato, Private Bag 3105, Hamilton 3240, New Zealand

Tel.: +64-7-8384834

Fax: +64-7-8384835

E-mail: mtwilson@waikato.ac.nz

P. W. Brownjohn · J. Shemmell

School of Physical Education, University of Otago, P.O. Box 56, Dunedin 9054, New Zealand

J. N. J. Reynolds

Department of Anatomy, University of Otago School of Medical Sciences, P.O. Box 913, Dunedin 9054, New Zealand 


\section{Introduction}

Transcranial magnetic stimulation (TMS) is a non-invasive technique for modulating brain function in humans. Short pulses of magnetic field are applied to the cortex with current-carrying coils. The rapidly changing magnetic field induces electrical currents within the cortex and stimulates activity over a wide area (a few $\mathrm{cm}^{2}$ ) within the brain [50]. TMS has had various reports of success in rehabilitation of stroke patients and in treating Parkinson's disease [35, 15, 10]; however, a recent study on rehabilitation of stroke patients did not find any clinical significance in applying TMS as part of treatment [47]. TMS has also been applied in slow wave sleep to increase the numbers of slow waves occuring, and while a useful tool for probing the structure of sleep [28] it is unclear as to whether TMS has any clinical significance with regard treatment of sleep disorders [6].

The effectiveness of the various protocols and the mechanisms by which plasticity mechanisms are engaged by TMS are poorly understood. The lack of underpinning science has limited the development and optimization of effective therapies [35]. For example, Hamada et al. have recently shown results that cast some doubt on previously accepted outcomes, meaning that an effective model is much needed to help clarify TMS effects [16]. Additionally, the potential for repetitive TMS to produce seizures has meant that it has been applied cautiously in humans. Coupled with a lack of animal studies this means that the full scope of its possibilities has not been investigated [31].

The modelling of brain dynamics using a field-based approach is now well established. Neural behaviour is modelled in terms of average firing rates and axonal flux rates of populations of neurons, rather than in terms of the behaviours of individual neurons $[51,30,9,19,40,4,46,7,37,52]$. This approach is particularly suited to the modelling of large-scale measures of brain activity, such as the electroencephalogram (EEG), which involve the sampling of many neurons. By the same token, the method will be appropriate to cases where large numbers of neurons are stimulated simultaneously, as in TMS. Recently, Robinson [36] has assembled a simple numerical model of spike-timing dependent plasticity (STDP) $[1,20,13$, 14] within neurons that builds upon the platform of neural field modelling. This allowed a first application of a neural field model to TMS. A single population of excitatory cells was considered, driven by external input from the TMS. Robinson showed that the net effect of the plasticity changes could be either the potentiation or depression of synapses depending upon the frequency of the applied stimulation. This model of plasticity was expanded by Fung et al. [11] to consider both inhibitory and excitatory populations and the resulting dynamics of synaptic weight changes. The behaviour of a system is governed by a saddle-node bifurcation and gamma resonance, which can lead to either stable or unstable synaptic changes depending on the form of the STDP model. The modelling suggests that a cortical system can self-organize into a position close to instability, leading to a richness in potential neural dynamics $[3,2,53]$.

In the current work we use the foundation provided by Robinson [36] and Fung et al. [11] to model changes in plasticity due to application of TMS pulses. Specifically, we model the application of different TMS protocols and compare the model results with experimental results. Moreover, the modelling allows us to make predictions about how to optimize existing TMS schemes that are experimentally verifiable. Our intention is to emphasize the possibilities of such a modelling ap- 
proach to further the understanding of TMS effects and highlight the difficulties and problems involved. We begin by outlining some of the key protocols of TMS. The modelling methodology is then discussed. Finally, we describe the application of the model to various experimental protocols and discuss the results in terms of current understanding. Suggestions for future experimental work are made.

\subsection{Repetitive TMS and theta burst stimulation}

Repetitive TMS (rTMS) is a class of protocols in which repeating series of stimuli are applied. There is a wide range of possibilities - for example application of single pulses at a constant rate (typically of order $1-10 \mathrm{~Hz}$ ) or application of bursts of pulses. Accepted understanding from TMS experiments on humans is that repetition frequencies above about $5 \mathrm{~Hz}$ favour potentiation [33,8] (e.g. increased size of motor evoked potentials) whereas frequencies at $1 \mathrm{~Hz}$ or lower can lead to depression $[49,5,8]$; the latter may be linked to synaptic downscaling in slow wave sleep $[28,48]$.

A commonly used bursting protocol is theta-burst stimulation (TBS) $[17,18]$. Here, trains of pulses are repeated at a theta band frequency, typically around $5 \mathrm{~Hz}$. A specific example is that suggested by Huang et al. [18], in which bursts of three pulses, $0.02 \mathrm{~s}$ apart, are repeated every $0.2 \mathrm{~s}$ (i.e. $5 \mathrm{~Hz}$ ). This is shown in Fig. 1a. It is possible that TMS may be more facilitatory if applied intermittently and inhibitory if applied continuously [41]. The reasons for this are unclear. It has been hypothesized that facilitory effects are produced more quickly than inhibitory effects, but the latter will eventually dominate. Therefore an intermittent burst allows time for inhibitory effects to decay away between bursts [18]. The timing of so-called indirect waves (I-waves) of activity may also be important; these are waves of activity caused by synaptic activation that occur after direct activation of axons [42]. For example continuous TBS depresses early I-waves [25] but intermittent TBS enhances later I-waves [24]. More recently, Hamada et al. [16] have suggested that the population of neurons that is stimulated is of importance since this may influence which form of I-waves predominates. A specific case of an intermittent protocol is intermittent theta-burst stimulation (iTBS). Bursts of pulses are applied for a period (e.g. $2 \mathrm{~s}$ ), followed by a quiet time (e.g. $8 \mathrm{~s})$, before another period of pulses. This contrasts with the continuous TBS (cTBS) protocol. The protocols are shown in Fig. 1. Panels (a) and (b) show cTBS and panel (c) shows iTBS. The delivery of an iTBS protocol is extended in time; a cTBS protocol may last $40 \mathrm{~s}$ (600 pulses) wheras the same number of pulses in iTBS would last $200 \mathrm{~s}$. Huang et al. [18] found that iTBS led to an increased MEP amplitude whereas cTBS at the same frequency led to a decrease in motor evoked potential (MEP) amplitude. Changes persisted for around one hour after treatment.

\subsection{Acute effects of TMS}

Acute effects can be probed with the paired-pulse approach. Two stimuli are given, a short period apart (typically $0-20 \mathrm{~ms}$ ) $[15,42,54]$, over the motor cortex. Often, the positioning of the TMS coil is such that an electromyogram (EMG) response can be detected at the wrist. The first stimulus (conditioning stimulus) is given 


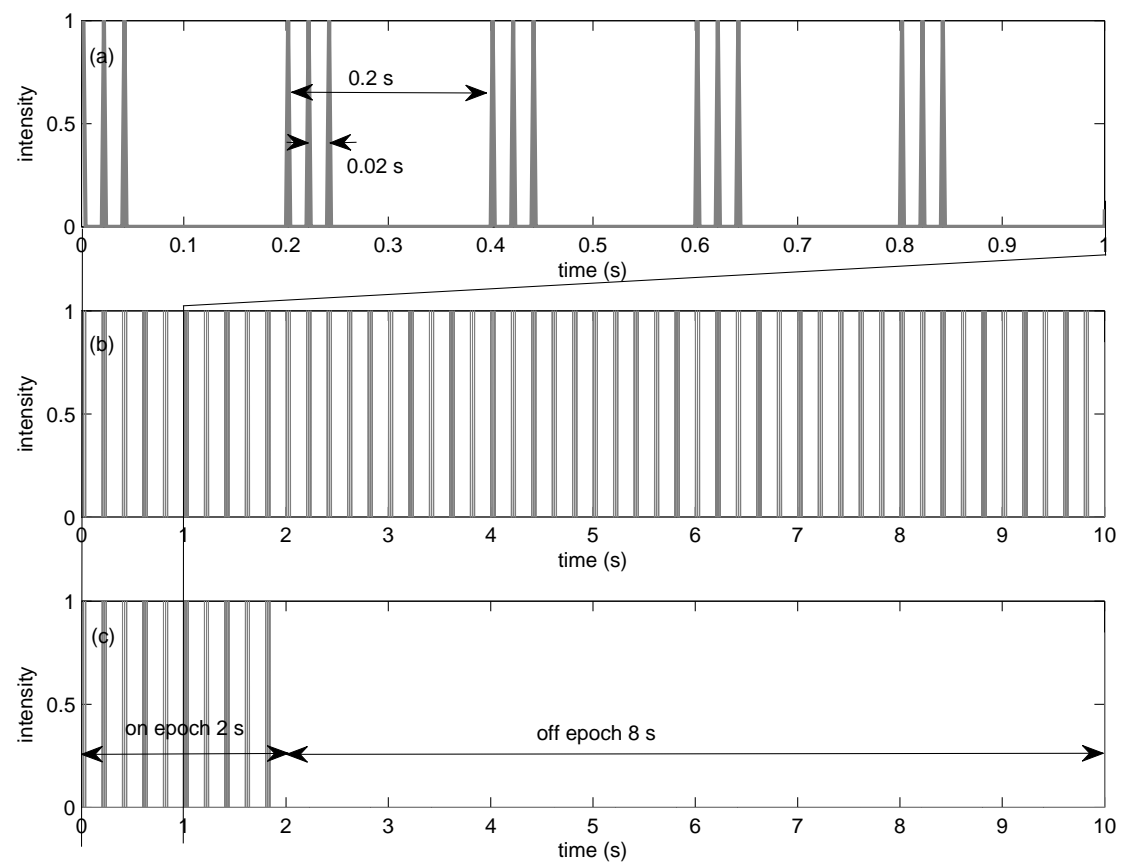

Fig. 1 Theta Burst Stimulation protocols. (a) A close-up of the TBS stimulation protocol in which bursts of three pulses are repeated five times a second. (b) The cTBS paradigm in which the bursts are applied repetitively (c) The iTBS paradigm in which bursts are applied for $2 \mathrm{~s}$, followed by an absence of bursts for $8 \mathrm{~s}$.

below motor-threshold level, so that while cortical activity is stimulated, there is no MEP produced. The effect of the second stimulus (test stimulus), which is applied above the motor-threshold level, depends upon the period between them. At short time intervals (around $5 \mathrm{~ms}$ or lower) there is short-latency intracortical inhibition (SICI) and the EMG detected at the wrist is decreased from the case of the test stimulus being applied alone [22]. This may be an effect of the GABA neurotransmitter [23]. At longer intervals (5 ms to order $40 \mathrm{~ms}$ ) the EMG is boosted. At longer intervals still (of order $40 \mathrm{~ms}$ or longer), there may be longlatency intracortical inhibition (LICI) and the EMG is again suppressed [29].

\section{Method}

We base this work upon the well-documented mean-field theory of Robinson and co-workers $[40,39,7,37]$. Rather than modeling individual neurons, we consider averaging properties over local groups of neurons. Key variables are then the mean firing-rate of type $a$ cells, $Q_{a}$, which is a function of the mean membrane potential $V_{a}$, and the mean spike rate in axons from type $b$ cells to type $a$ cells, $\phi_{a b}$. Here 
the subscripts $a$ and $b$ can be assigned either ' $e$ ' to denote excitatory cells, or ' $i$ ' to denote inhibitory cells.

Changes in firing rate are modelled as a linear response to the incoming input from other cells $[39,40,21]$. We therefore concern ourselves with changes in firing rate and spike rate from their equilibrium values. Writing $Q_{a}(t)=Q_{a}^{0}+\delta Q_{a}(t)$ and $\phi_{a b}(t)=\phi_{a b}^{0}+\delta \phi_{a b}(t)$, where the superscript ' 0 ' denotes the steady-state value of the variable, we obtain a set of linearized equations for the fluctuations about the equilibrium values.

The first of these describes the firing rate in terms of the axonal input:

$$
\delta Q_{a}(t)=\int_{t^{\prime}=-\infty}^{\infty} \sum_{b} G_{a b} L_{a b}\left(t-t^{\prime}\right)\left[\delta \phi_{a b}\left(t^{\prime}\right)+\delta \phi_{a b}^{\mathrm{ext}}\left(t^{\prime}\right)\right] d t^{\prime}
$$

where $L_{a b}\left(t-t^{\prime}\right)$ is the impulse response function of soma potential of cells of type $a$ in response to axonal input at $t=t^{\prime}, G_{a b}$ shows the gain of the system, and $\delta \phi_{a b}^{\text {ext }}(t)$ is a rate of axonal firing events from cells of type $b$ to those of type $a$ induced by the application of the external input, namely the TMS pulses and noise. While there are several mechanisms by which excitation may occur during application of TMS, axonal stimulation is a major contributor [42,44]. Excitation of cell bodies may also occur. While it would be straightforward to include it within the modelling scheme, we do not do so in this work in order to restrict the number of parameters required.

The gain $G_{a b}$ is given by:

$$
G_{a b}=\left.\frac{\partial Q_{a}}{\partial V_{a}}\right|_{\text {eqm }} N_{a b} s_{a b},
$$

where $N_{a b}$ and $s_{a b}$ are the mean number and strength repectively of the connections from neurons of type $b$ to those of type $a$. The strength $s_{a b}$ represents the time-integral of the post-synaptic potentials and is the voltage change at the soma per unit firing rate at a synapse. Since TMS is not particularly well localized compared with typical lengths for intra-cortical connections we consider only spatially symmetric changes so that the variables are functions of time only, but not space. This has the advantage of simplifying the numerical modelling. The spatially symmetric model is valid for long wavelength modes of stimulation such as the biphasic waveform typically used in repetitive TMS protocols. However, the model is generalizable to spatially varying states [11]. The model of reference [37] includes feedback delayed in time as a simple model of a thalamocortical loop. For the purposes of simplifying the analysis, we do not consider this here, and confine ourselves to intra-cortical interactions only.

The second equation describes propagation of spikes along axons with a second impulse response function. We have:

$$
\delta \phi_{a b}(t)=\int_{t^{\prime}=-\infty}^{\infty} \Gamma_{a b}\left(t-t^{\prime}\right) \delta Q_{b}\left(t^{\prime}\right) d t^{\prime}
$$

where the impulse response function $\Gamma_{a b}\left(t-t^{\prime}\right)$ describes the response of the axonal firing rate to changes in firing rate $\delta Q_{b}\left(t^{\prime}\right)$. 
It is instructive to write the linearized equations in fourier space. We assume the variable $x(t)$ repeats over a time-scale $T$ (a form suitable for modelling repeating stimulation such as in repetitive TMS) and write it in terms of its fourier components $\tilde{x}(\omega)$ as:

$$
x(t)=\sum_{\omega} \tilde{x}(\omega) e^{i \omega t}
$$

where the sum is taken over angular frequencies $\omega=2 \pi n / T$ where $n$ is an integer, so that

$$
\tilde{x}(\omega)=\frac{1}{T} \int_{0}^{T} x(t) e^{-i \omega t} d t .
$$

This definition is convenient for the analysis of trains of TMS pulses that have a defined repetition time, and means that $x(t)$ and $\tilde{x}(\omega)$ carry the same dimensions. The impulse response functions $L_{a b}$ and $\Gamma_{a b}$ simply map to transfer functions. Limiting the integrals in Eqs. (1) and (3) to a time range of $-T / 2$ to $T / 2$, which is reasonable if $T$ is much larger than timescales for $L_{a b}$ and $\Gamma_{a b}$, we obtain from Eqs. (1) and (3):

$$
\delta \tilde{Q}_{a}(\omega)=T \sum_{b} G_{a b} \tilde{L}_{a b}(\omega)\left[\delta \tilde{\phi}_{a b}(\omega)+\delta \tilde{\phi}_{a b}^{\text {ext }}(\omega)\right]
$$

and

$$
\delta \tilde{\phi}_{a b}(\omega)=T \tilde{\Gamma}_{a b}(\omega) \delta \tilde{Q}_{b}(\omega)
$$

respectively. In these equations $\tilde{x}(\omega)$ denotes the fourier transform of $x(t)$. For notational convenience, we now drop the tilde mark on the fourier transform.

The transfer functions $L_{a b}(\omega)$ and $\Gamma_{a b}(\omega)$ can be written in Fourier space as follows. The synaptic and soma response is typically described by a bi-exponential function $[39,40,21,7]$. For the case of excitatory synapses (i.e. $b=e$ ) we write:

$$
L_{a e}(\omega)=\frac{1}{T\left(1+\frac{i \omega}{\alpha_{a e}}\right)\left(1+\frac{i \omega}{\beta_{a e}}\right)} .
$$

where the rate constants $\alpha_{a e}$ and $\beta_{a e}$ describe the rates of growth and decay of the soma-response to incoming axonal input. This function incorporates the timecourse of the excitatory post-synaptic potential (EPSP). However, for the case of inhibitory synapses $(b=i)$ we include the effects of both $\mathrm{GABA}_{\mathrm{A}}$ and the much longer lasting (several hundred milliseconds $[32,34]$ ) GABA $_{\mathrm{B}}$ neurostransmitters. Therefore we use a combination of two of these functions for the inhibitory postsynaptic potential (IPSP):

$$
L_{a i}(\omega)=\frac{1}{2 T\left(1+\frac{i \omega}{\alpha_{a i, A}}\right)\left(1+\frac{i \omega}{\beta_{a i, A}}\right)}+\frac{1}{2 T\left(1+\frac{i \omega}{\alpha_{a i, B}}\right)\left(1+\frac{i \omega}{\beta_{a i, B}}\right)}
$$

where the $A$ and $B$ subscripts denote the response to $\mathrm{GABA}_{\mathrm{A}}$ and $\mathrm{GABA}_{\mathrm{B}}$ respecively.

The axonal transfer function can be modelled with a damped wave equation [19, $40,26,7]$; we use the form of Robinson et al. [40], leading to:

$$
\Gamma_{a b}(\omega)=\frac{1}{T\left(1+\frac{i \omega}{\gamma_{a b}}\right)^{2}},
$$


where $\gamma_{a b}$ are axonal rate constants.

Solving Eqs. (6) and (7) for the firing-rate responses $\delta Q_{b}(\omega)$ to external axonal input $\delta \phi_{a b}^{\text {ext }}$ is now straightforward. We have the matrix equation for the excitatory and inhibitory response:

$$
\mathbf{Q}(\omega)=M(\omega) \mathbf{Q}(\omega)+\mathbf{X}(\omega)
$$

where

$$
\mathbf{Q}(\omega)=\left(\begin{array}{l}
\delta Q_{e}(\omega) \\
\delta Q_{i}(\omega)
\end{array}\right)
$$

the dimensionless matrix $M$ is given by:

$$
M(\omega)=T^{2}\left(\begin{array}{ll}
G_{e e} L_{e e} \Gamma_{e e}(\omega) & G_{e i} L_{e i}(\omega) \Gamma_{e i}(\omega) \\
G_{i e} L_{i e}(\omega) \Gamma_{i e}(\omega) & G_{i i} L_{i i}(\omega) \Gamma_{i i}(\omega)
\end{array}\right)
$$

and $\mathbf{X}(\omega)$, with dimensions of inverse time, is given by:

$$
\mathbf{X}(\omega)=T\left(\begin{array}{l}
G_{e e} L_{e e}(\omega) \delta \phi_{e e}^{\mathrm{ext}}(\omega)+G_{e i} L_{e i}(\omega) \delta \phi_{e i}^{\mathrm{ext}}(\omega) \\
G_{i e} L_{i e}(\omega) \delta \phi_{i e}^{\mathrm{ext}}(\omega)+G_{i i} L_{i i}(\omega) \delta \phi_{i i}^{\mathrm{ext}}(\omega)
\end{array}\right)
$$

One can solve for $\mathbf{Q}(\omega)$ as:

$$
\mathbf{Q}(\omega)=(I-M(\omega))^{-1} \mathbf{X}(\omega)
$$

where $I$ is the identity matrix. This gives the response in firing rates of the excitatory and inhibitory populations to external drive of the axons caused by TMS impulses.

To this point, we have kept the mathematical presentations as general as possible. However, in the modelling we simplify the situation by considering that: (a) the response function $L_{a b}$ is a function of the pre-synaptic cell $b$ only, that is $\alpha_{e e}=\alpha_{i e}=\alpha_{e} ; \beta_{e e}=\beta_{i e}=\beta_{e} ; \alpha_{e i, A}=\alpha_{i i, A}=\alpha_{A} ; \beta_{e i, A}=\beta_{i i, A}=\beta_{A}$; $\alpha_{e i, B}=\alpha_{i i, B}=\alpha_{B} ; \beta_{e i, B}=\beta_{i i, B}=\beta_{B}$; (b) the propagation of signals along axons is a function of the pre-synaptic cell $b$ only, that is $\gamma_{e e}=\gamma_{i e}=\gamma_{e}$; $\gamma_{e i}=\gamma_{i i}=\gamma_{i}$; and (c) that the connections between cells are random (i.e. that the number of connections from cells of type $b$ to a given cell of type $a$ is proportional to the number of $b$-type cells) so that $G_{e e}=G_{i e}=G_{e}$ and $G_{e i}=G_{i i}=G_{i}$.

\subsection{Stability}

Equation (15) predicts that the system will be unstable when $\operatorname{det}[I-M(\omega)]=0$. Since $M$ is a $2 \times 2$ matrix and the transfer functions $L_{a b}(\omega)$ and $\Gamma_{a b}(\omega)$ are analytic functions of $\omega$ it is straightforward to write down an equation for $\omega$ such that this condition is obeyed. In the numerical modelling, we use symbolic algebra within MATLAB to turn this equation into a polynomial in $\omega$ which is then solved for $\omega$. The system will be stable so long as the imaginary parts of the roots are positive. Thus the stability of any set of modelled conditions can be tested. We confine ourselves to situations that are stable. 


\subsection{The effect of magnetic fields on neurons}

We now consider the effect of a TMS pulse on the cortical system. We assume that a pulse creates a short-lived excitation of the axons of stimulated neurons [42]. We model this as an increased axonal firing rate. TMS pulses are typically very short in duration (around $0.2 \mathrm{~ms}[35]$ ) and we make the assumption that the excitation occurs over a time-scale that is shorter than the EPSP and IPSP events, so that it can be modelled as a delta-function impulse.

The mechanisms by which excitation of the cortex occurs under a TMS pulse are still poorly understood [16], and are likely to vary with intensity of the pulse [42]. However, it is often assumed that high intensities produce excitation on excitatory axonal inputs to excitatory synapses $[42,44]$. We therefore specifically include the excitation of different axonal populations in the model. We write the input functions as $\phi_{e e}^{\text {ext }}(t)=\lambda_{e e} \phi^{\mathrm{TMS}}(t), \phi_{i e}^{\text {ext }}(t)=\lambda_{i e} \phi^{\mathrm{TMS}}(t)$ and $\phi_{e i}^{\text {ext }}=\phi_{i i}^{\text {ext }}=0$ where $\phi^{\mathrm{TMS}}(t)$ is a series of delta-functions describing the repetitive train of applied pulses:

$$
\phi^{\mathrm{TMS}}(t)=\sum_{i} \delta\left(t-t_{i}^{p}\right)-\left\langle\sum_{i} \delta\left(t-t_{i}^{p}\right)\right\rangle_{t}
$$

where $t_{i}^{p}$ is the time of the $i$-th pulse of the TMS protocol. This function gives an instantaneous increase in axonal flux at each pulse of the TMS protocol. The subtraction of the mean (which is equal to the average applied pulse rate) ensures that $\phi^{\mathrm{TMS}}(t)$ averages to zero. The $\lambda_{e e}$ and $\lambda_{i e}$ are then parameters that can be specified in the modelling. It is likely that the stimulated populations, and therefore $\lambda_{e e}$ and $\lambda_{i e}$, vary with intensity of the pulse and the orientation of the applied current $[41,16]$. We acknowledge that the most appropriate form of the $\delta \phi_{a b}^{\text {ext }}(t)$ is very uncertain; however we believe that Eqs. (16) modulated by $\lambda_{e e}$ and $\lambda_{i e}$ is a reasonable approach for the purposes of illustrating neural field theory applied to plastic changes caused by TMS.

Since the $\delta \phi_{e e}^{\text {ext }}(t)$ and $\delta \phi_{i e}^{\text {ext }}(t)$ are both proportional to the same function $\phi^{\mathrm{TMS}}(t)$, and $M(\omega)$ is a $2 \times 2$ matrix, we can explicitly write down $Q_{e}(\omega)$ from Eq. (15) as:

$$
\begin{array}{r}
Q_{e}(\omega)=\left[\frac{\lambda_{e e}+\left(\lambda_{i e}-\lambda_{e e}\right) T^{2} G_{i} L_{i}(\omega) \Gamma_{i}(\omega)}{1-T^{2} G_{e} L_{e}(\omega) \Gamma_{e}(\omega)-T^{2} G_{i} L_{i}(\omega) \Gamma_{i}(\omega)}\right] G_{e} L_{e}(\omega) \delta \phi^{\mathrm{TMS}}(\omega) \\
=Q_{e}^{\mathrm{T}}(\omega) \delta \phi^{\mathrm{TMS}}(\omega)
\end{array}
$$

where $Q_{e}^{T}(\omega)$ is a transfer function.

\subsection{Changes in synaptic weights}

A key stage in the modelling process is the evaluation of the changes in synaptic weights occuring as a result of the TMS input. We use the phenomonological STDP approach. The change in synaptic weight between neurons is dependent upon the difference in time between the post-synaptic and pre-synaptic spikes. Robinson [36] and Fung et al. [11] have demonstrated how STDP can be captured in a neural field scheme. While the validity of a simple formulation of STDP is still a matter of some debate $[43,27]$ it leads to a straightforward modelling scheme. An 
alternative approach has been taken by Fung and Robinson [12] in which calcium dependent plasticity has been modelled.

In the field approach, the change in average normalized weight $w_{a b}\left(w_{a b}(t=\right.$ $0)=1$ ) for connections from neurons of type $b$ to those of type $a$ is dependent upon the correlation between the incoming synaptic flux $\delta \phi_{a b}(t)$ and the outgoing firing rate $\delta Q_{a}(t)$ :

$$
\frac{d w_{a b}(t)}{d t}=\int_{\tau=-T / 2}^{T / 2}\left\langle\delta Q_{a}\left(t^{\prime}+\tau\right) H_{a b}(\tau) \delta \phi_{a b}\left(t^{\prime}\right)\right\rangle_{t^{\prime}} d \tau
$$

where $T$ is a suitably chosen timescale, the angle brackets show a temporal average over $t^{\prime}$ centred around time $t$, and $H_{a b}(\tau)$ describes the STDP function, fitted with two exponentials which depend on the post-synaptic neuron $[1,20,13,14]$ :

$$
H_{a b}(\tau)=\left\{\begin{array}{l}
A_{a}^{+} e^{-\tau / t_{a}^{+}}, \tau>0 \\
A_{a}^{-} e^{\tau / t_{a}^{-}}, \quad \tau \leq 0
\end{array}\right.
$$

where $A_{a}^{+}$and $A_{a}^{-}$are dimensionless. Physically, the integral in Eq. (18) consists of the correlation in time between the incoming flux rate $\phi_{a b}$ and the firing rate $Q_{a}$ a time $\tau$ later, weighted by the STDP function $H_{a b}(\tau)$ and integrated over $\tau$.

At this point we must make some comment on the selection of timescales in Eq. (18). The STDP function $H_{a b}(\tau)$ has two time-constants, $t_{a}^{+}$and $t_{a}^{-}$, which are of the order of a few tens of milliseconds. The integral over $\tau$ therefore needs to be taken over a time-period $T$ that is rather larger than this in order to encapsulate the full scope of the exponential decays. The averaging over time $t^{\prime}$ that is represented in the angle brackets must also be done over a time-scale larger than $t_{a}^{+}$and $t_{a}^{-}$, but smaller than the time-scales for changes in the synaptic weights themselves (of order a few seconds) so that $w_{a b}$ can be considered constant across the period of the integration. One may use the same period $T$ for both integrals over $\tau$ and $t^{\prime}$, if $T$ is between a few tens of milliseconds and a few seconds in length. We also note that a few seconds is the typical scale over which a repetitive TMS protocol repeats, and therefore we can reasonably and conveniently use the same timescale $T$ for the repetition period of the TMS driving $\delta \phi^{\mathrm{TMS}}(t)$.

\subsection{Interpretation in Fourier Domain}

The plasticity integral Eq. (18) can be evaluated with fourier methods. Writing $\delta Q_{b}, H_{a b}$ and $\delta \phi_{a b}$ in terms of their fourier components, we obtain [11]:

$$
\frac{d w_{a b}(t)}{d t}=T \sum_{\omega} \delta Q_{b}^{*}(\omega) \delta \phi_{a b}(\omega) H_{a b}(\omega)
$$

where $H(\omega)$ is given by:

$$
H_{a b}(\omega)=\frac{A_{a}^{+}}{T}\left[\frac{t_{a}^{+}-i \omega t_{a}^{+}}{1+\omega^{2}\left(t_{a}^{+}\right)^{2}}\right]+\frac{A_{a}^{-}}{T}\left[\frac{t_{a}^{-}+i \omega t_{a}^{-}}{1+\omega^{2}\left(t_{a}^{-}\right)^{2}}\right] .
$$


Furthermore, we can write $\delta \phi_{a b}$ in terms of the change in firing rate $\delta Q_{b}$ and the transfer function $\Gamma_{a b}$, through Eq. (7) giving:

$$
\begin{array}{r}
\frac{d w_{a b}(t)}{d t}=T^{2} \sum_{\omega}\left|\delta Q_{b}(\omega)\right|^{2} \Gamma_{a b}(\omega) H_{a b}(\omega) \\
=T^{2} \sum_{\omega}\left|\delta Q_{b}(\omega)\right|^{2} \operatorname{Re}\left[\Gamma_{a b}(\omega) H_{a b}(\omega)\right]
\end{array}
$$

where the latter follows from $\Gamma_{a b}(-\omega)=\Gamma_{a b}^{*}(\omega)$ and $H_{a b}(-\omega)=H_{a b}^{*}(\omega)$ since $\Gamma_{a b}(t)$ and $H_{a b}(t)$ are real functions of time. In this work we concentrate on the growth in strength between the $e$ and $e$ connections, which predominate in the cortex, that is $d w_{e e}(t) / d t$, given by:

$$
\frac{d w_{e e}(t)}{d t}=T^{2} \sum_{\omega}\left|\delta Q_{e}(\omega)\right|^{2} \operatorname{Re}\left[\Gamma_{e}(\omega) H_{e e}(\omega)\right] .
$$

This is simply a weighted sum over angular frequency of the power in the fluctuations in firing rate $\delta Q_{e}(\omega)$. One therefore has a straightforward recipe for evaluating the rate of change of synaptic weights. Given an external stimulus function due to the TMS machine $\delta \phi^{\mathrm{TMS}}(t)$, that repeats over a period $T$, we first construct the axonal stimulation functions $\delta \phi_{a b}^{\text {ext }}(t)$ from Eq. (16) and find their fourier components $\delta \phi_{a b}^{\text {ext }}(\omega)$. Then one can construct the matrix $\mathbf{M}(\omega)$ and vector $\mathbf{X}(\omega)$ and solve Eq. (15) for the response $\delta Q_{e}(\omega)$ of the excitatory population to the external stimulus. Then Eq. (23) yields the overall rate of change of the synaptic weight $w_{e e}$ to the stimulus. A summary of the system showing the variables and transfer functions is shown in Fig. 2.

In what follows, we use a rate of change of synaptic weight normalized per pulse of the TMS input. This allows us to compare protocols that have a different repetition rate but equal numbers of pulses (e.g. cTBS and iTBS) on an equal basis. If a sequence repeats every time $T$, and contains $N_{T}$ pulses in this time (that is, an average of $N_{T} / T$ pulses per unit time), then we divide Eq. (23) by $N_{T} / T$ to obtain the change in weight per TMS pulse $\Delta w_{e e}$ :

$$
\Delta w_{e e}=\frac{T^{3}}{N_{T}} \sum_{\omega}\left|\delta Q_{e}(\omega)\right|^{2} \operatorname{Re}\left[\Gamma_{e}(\omega) H_{e e}(\omega)\right] .
$$

We apply Eq. (24) to calculate the response per pulse of an applied TMS protocol. Note that this quantity does not scale with $T$ since $\Gamma_{e}$ and $H_{e e}$ both scale as $1 / T$ and $N_{T}$ scales as $T$.

A key assumption in the above analysis is that changes in synaptic efficiency do not feedback into changes in the response of the neural populations to stimulation. In other words, Eq. (24) requires that plasticity changes are small. However, once can feed back the changes in synaptic weight in a simple way by splitting the time sequence of pulses into a number of shorter epochs, and evaluating the average change in $w_{e e}$ within each epoch, then modifying $G_{e}$ for the calculation of $Q_{e}(\omega)$ and $d w_{e e} / d t$ for the next epoch. This is at the expense of increased computational time and the loss of linearity in the model. To do this, we use 600 epochs (which means that there is on average one pulse per epoch for the cTBS and iTBS protocols), and modify $G_{e}$ after every epoch:

$$
G_{e}(t+\delta t)=G_{e}(t)\left[1+\Delta w_{e e}(t)\right]
$$




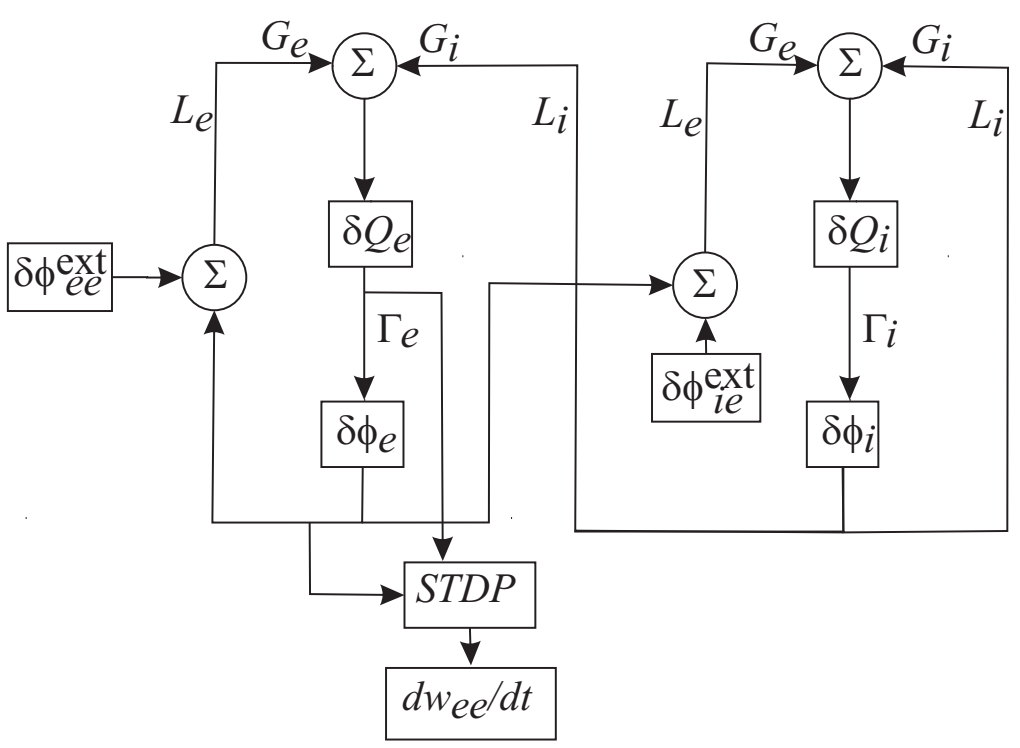

Fig. 2 A summary of the numerical model. Excitatory cells are on the left-hand side; inhibitory cells on the right. The system is driving by the inputs $\delta \phi_{e e}^{\text {ext }}$ and $\delta \phi_{i e}^{\text {ext }}$. The variables $\delta Q_{e}$ (fluctuations in mean excitatory firing rate) and $\delta \phi_{e}$ (fluctuations in excitatory axonal event rate) are combined through the STDP formalism to evaluate the rate of change of weight, $d w_{e e} / d t$. The functions $L_{e}$ and $L_{i}$ denote the excitatory and inhibitory axonal transfer functions respectively, and $\Gamma_{e}$ and $\Gamma_{i}$ denote respectively the excitatory and inhibitory cell responses to synaptic input. The parameters $G_{e}$ and $G_{i}$ are respectively the excitatory and inhibitory gains of the feedback loops. The symbol $\Sigma$ denotes a summation.

where $\Delta w_{e e}$ is calculated with Eq. (24) and $\delta t=T / N_{T}$, the average time per pulse of the protocol. In order to keep the simplifying symmetry between $G_{e e}$ and $G_{i e}$ we modify both weights in this way. More realistically, however, all weights $G_{e e}$, $G_{i e}, G_{e i}$ and $G_{i i}$ would need to be modified separately.

There are many parameters in this model, but many are constrained physically. Table 1 gives a list of standard parameters chosen for this modelling. Values for the mean-field cortical modelling have been chosen drawing from references $[38,39,37$, $11,45,46]$, with smaller rates being used for $\alpha_{B}$ and $\beta_{B}$ to model the long timescale GABA $_{B}$ inhibitory post-synaptic potentials $[32,34]$. Values for the STDP have been chosen with reference to [1] and [11]. The model is particularly sensitive to the STDP parameters, as elucidated in the results below. We confine ourselves to the case where $A_{e}^{-}$is negative, so that a post-synaptic event occuring before a pre-synaptic event leads to a reduction in synaptic weight.

\section{Results and discussion}

\subsection{Continuous TMS protocols}

We start by considering the case of a continuous repetitive TMS protocol consisting of repetitive bursts of pulses, $0.02 \mathrm{~s}$ between pulses in each burst. We allow the time 
Table 1 Standard values for the model

\begin{tabular}{llll}
\hline Parameter & Description & Value & Unit \\
\hline$\alpha_{e}$ & Cell rise rate to excitatory input & 280 & $\mathrm{~s}^{-1}$ \\
$\beta_{e}$ & Cell fall rate to excitatory input & 70 & $\mathrm{~s}^{-1}$ \\
$\gamma_{e}$ & Excitatory axonal rate constant & 110 & $\mathrm{~s}^{-1}$ \\
$\alpha_{\mathrm{A}}$ & Cell rise rate to inhibitory GABA $\mathrm{A}$ input & 400 & $\mathrm{~s}^{-1}$ \\
$\beta_{\mathrm{A}}$ & Cell fall rate to inhibitory GABA $\mathrm{Anput}$ & 100 & $\mathrm{~s}^{-1}$ \\
$\alpha_{\mathrm{B}}$ & Cell rise rate to inhibitory GABA $\mathrm{B}$ input & 20 & $\mathrm{~s}^{-1}$ \\
$\beta_{\mathrm{B}}$ & Cell fall rate to inhibitory GABA $\mathrm{B}$ input & 5 & $\mathrm{~s}^{-1}$ \\
$\gamma_{i}$ & Inhibitory axonal rate constant & 1000 & $\mathrm{~s}$ \\
$t_{a}^{+}$ & STDP positive decay constant & 0.020 & $\mathrm{~s}$ \\
$t_{a}^{-}$ & STDP negative decay constant & 0.020 & $\mathrm{~s}$ \\
$A_{e}^{+}$ & STDP positive weight constant & 1.0 & \\
$A_{e}^{-}$ & STDP negative weight constant & -0.75 & \\
$G_{e}$ & Excitatory gain & 0.8 & \\
$G_{i}$ & Inhibitory gain & -0.6 & \\
\hline
\end{tabular}

between bursts and the number of pulses per burst to vary. This encompasses a wide range of rTMS protocols, including those of single pulses applied repetitively (one pulse per burst) and also cTBS (three pulses per burst at a $5 \mathrm{~Hz}$ burst rate). We consider stimulation of the excitatory inputs to the excitatory neurons, so that $\lambda_{e e}=1.0$, and $\lambda_{i e}=0.0$. Results are shown in Fig. 3a as a contour plot of $\Delta w_{e e}$ per pulse against burst frequency (y-axis) and pulses per burst assuming that the changes in weight are small. The colour indicates the size of $\Delta w_{e e}$. The $\Delta w_{e e}=0$ contour is shown by a thick dashed line. The solid line shows the limit of possibility for this protocol - above the line the bursts are too frequent to accommodate the number of pulses required in each burst. On the line itself, there are no gaps between the bursts, and the system corresponds to a repetitive TMS protocol at $50 \mathrm{~Hz}$. One can see that predominantly the result is negative, denoting a reduction in the strength of synapses. In particular, we see that for the case of 1 pulse per burst (y-axis), $\Delta w_{e e}<0$. There is a region where the results can be strongly positive, at high numbers of pulses per burst and low burst rates. This again agrees with experiment where high frequency rTMS (in this case $50 \mathrm{~Hz}$ ) can give strong potentiation [8]. Recalling that the figure shows $\Delta w_{e e}$ per pulse, and that trains of TMS are typically several hundred pulses long, in the region of high potentiation we can see that total potentiation can be significantly greater than 1 . This would correspond to a large increase in the weight of an excitatory-to-excitatory synapse. In practice, such a high response would not occur, but the modelling does suggest that a strong increment in synaptic weight is possible. This may be connected with the risk of seizure when using TMS at high pulse rates for an extended time. The specific result for cTBS can be found from the plot at the position denoting 3 pulses per burst at $5 \mathrm{~Hz}$. In this case it is negative $\left(-17.6 \times 10^{-3}\right)$ agreeing with Huang et al. [18]. Multiplying by 600 for a typical cTBS sequence gives a total change in weight of 10.6, which is substantial. However, it is unclear as to how large $\lambda_{e e}$ and $\lambda_{i e}$ should be. In most of the modelling presented here, we use a sum of 1.0, indicating that each TMS pulse corresponds to a re-timing of a single firing event in each excitatory-to-excitatory axon. Since intracellular recording during TMS has not been carried out, it is difficult to know how realistic this assumption is. 

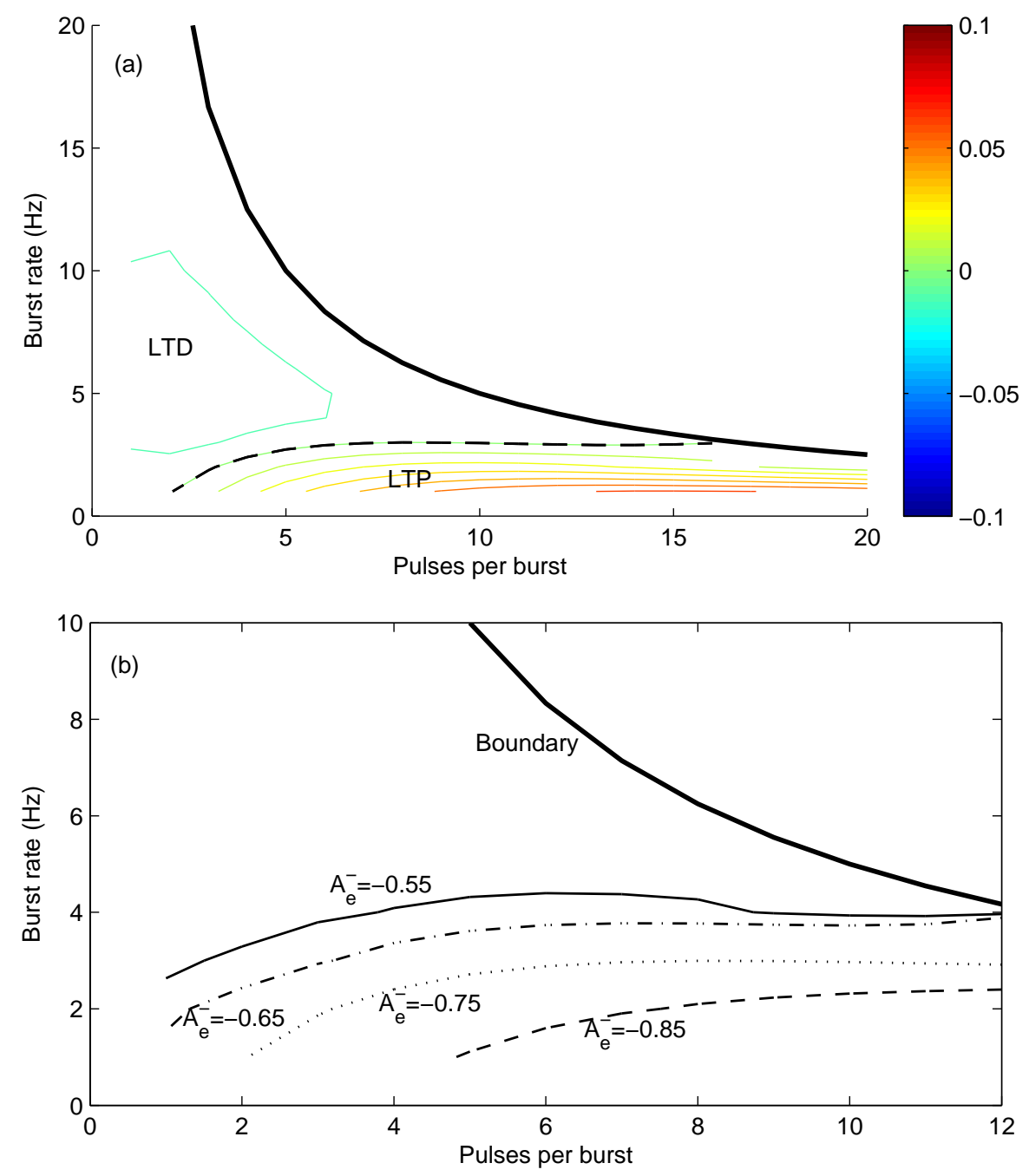

Fig. 3 (a) A plot of $\Delta w_{e e}$ per pulse, from Eq. (24) for continuous TMS protocols. Results are presented as a contour plot of $\Delta w_{e e}$ per pulse as a function of $50 \mathrm{~Hz}$ pulses per burst (x-axis) against burst rate (y-axis). Key: LTD - Long term depression; LTP - Long term potentiation. (b) A plot of the position of the border between LTD and LTP as a function of $A_{e}^{-}$. The thick solid line shows the limit of the range of possible burst rates and pulses per burst. 


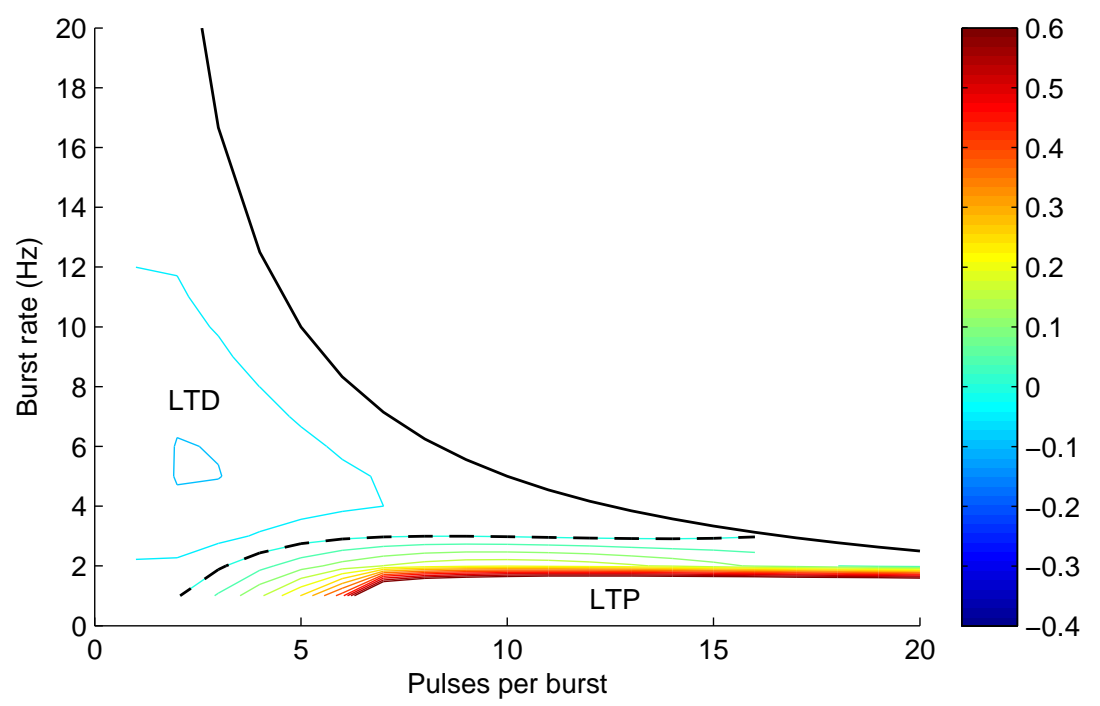

Fig. 4 A plot of the change in $w_{e e}$ over a 600 -pulse cTBS protocol as a function of $50 \mathrm{~Hz}$ pulses per burst (x-axis) against burst rate (y-axis). Key: LTD — Long term depression; LTP - Long term potentiation.

Results are particularly sensitive to the STDP parameters. We show this by looking at the variation in results for the example of changes in $A_{e}^{-}$. Figure $3 \mathrm{~b}$ shows the zero contour (border between LTP and LTD) as a function of $A_{e}^{-}$, with other parameters remaining constant. A more negative $A_{e}^{-}$favours depression. When $A_{e}^{-}<-1.0$, there is no region of potentiation present.

Finally for the continuous protocol, the effect of the feed back of synaptic weight into the calculation of $Q_{e}(\omega)$ is shown. Results are presented in Fig. 4 as a plot of change in $w_{e e}\left(w_{e e}(t=0)=1\right)$ over the whole protocol, against burst frequency and pulses per burst. In this case $\lambda_{e e}=0.1$ and $\lambda_{i e}=0.0$; higher values result in unreasonable growth in weights. Results are similar to Fig. $3 \mathrm{a}$ in that the boundary between LTD and LTP remains the same. However, there is substantial growth in synaptic weight at low burst rates for more than two pulses per burst.

\subsection{Intermittent protocols}

Intermittent protocols are similar to the continuous ones described above, however the difference is that the pulse trains are applied for a given length of time, which is then followed by a quiet period, before the pulses are again repeated. The iTBS protocol of Huang et al. [18] shown in Fig. 1c is an example; three-pulse bursts, are repeated every $0.2 \mathrm{~s}$ for $2 \mathrm{~s}$, followed by a quiet period of $8 \mathrm{~s}$ in which no external stimulation is applied, before the next $2 \mathrm{~s}$ burst is applied. Figure $5 \mathrm{a}$ shows the results of such an intermittent protocol, with a $2 \mathrm{~s}$ epoch of pulses followed by an $8 \mathrm{~s}$ quiet time. Results are different from those of cTBS; specifically, the region of negative change in $\Delta w_{e e}$ has become smaller and is confined to small burst rates and small numbers of pulses per burst. The iTBS result is positive - with a value 
of $\Delta w_{e e}=+7.6 \times 10^{-3}$ per pulse. Again, results are sensitive to STDP parameters such as $A_{e}^{-}$. Figure $5 \mathrm{~b}$ shows how the zero contour (the border between LTP and LTD) changes with variations in $A_{e}^{-}$(other parameters remaining constant). For $A_{e}^{-}<-1.0$, there is no region of potentiation; for $A_{e}^{-}>-0.65$ there is complete potentiation in the intermittent protocol.

Finally for iTBS the case where the changes in weights are fed back into the calculation of $Q_{e}(\omega)$ is considered. Results are shown in Fig. 6 for the case of $\lambda_{e e}=0.1, \lambda_{i e}=0.0$. As for cTBS, the boundary between LTD and LTP remains the same. However, the LTP becomes very pronounced at high burst rates and high pulses per burst. Biophysically, it is likely that other mechanisms that are not modelled prevent such changes occuring.

The differences between the continuous and intermittent protocols can be elucidated by looking at the terms contributing to Eq. (24). Figure 7 shows the functions $\left|\phi^{\mathrm{TMS}}(f)\right|^{2}$ for the continuous (a) and intermittent (b) protocols, the modulus squared of the transfer function $\left|Q_{e}^{T}(f)\right|^{2}$ of Eq. (17) (c), and $\operatorname{Re}\left[\Gamma_{e}(f) H_{e e}(f)\right]$ (d), as functions of frequency $f$, where $f=\omega / 2 \pi$. The result of the integral in Eq. (24) is proportional to the sum over frequencies of the product of the functions shown in parts (a), (c) and (d), for the case of the continuous protocol, and (b), (c) and (d) for the case of the intermittent protocol. In order to produce a large effect, we require the spectrum of the stimulation (parts (a) and (b) for cTBS and iTBS respectively) to overlap well with the transfer function $Q_{e}^{T}(f)$ and the plasticity function $\operatorname{Re}\left[H_{e e}(f) \Gamma_{e}(f)\right]$. The intermittent stimulation has more power close to zero frequency, and therefore couples more positively with the transfer function than the continuous protocol. We remark that the exact form of $\left|Q_{e}^{T}(f)\right|^{2}$ is not of great importance to the final result, unless strong resonances are present. Therefore, we expect our model to be fairly insensitive to the modelling of the cortical dynamics, allowing other models such as that used by Bojak and Liley [4] or SteynRoss et al. [46] to be used without major impact on results. Of most importance in the cortical dynamics is the modelling of the excitatory axonal transfer function, $\Gamma_{e}(f)$ since this will control the shape of $\operatorname{Re}\left[H_{e e}(f) \Gamma_{e}(f)\right]$.

Where resonances exist in the transfer function $Q_{e}^{T}(f)$ it is logical to ask whether these can be exploited for application of TMS. The transfer function $Q_{e}^{T}(f)$ can be broadly related to the electroencephalogram (EEG) [7]. Experimentally, for example, Massimini et al. have shown that low frequency $(0.8 \mathrm{~Hz}) \mathrm{rTMS}$ can trigger slow wave oscillations during stage 2-4 sleep [28]. In other words, a natural resonance in the brain can easily be stimulated by applying TMS at the appropriate frequency. In this modelling we use the theta-resonance as an example. In a population of individuals, any theta-band resonances in the EEG will vary in frequency, and therefore one can consider application of TBS that is optimized to an individual's EEG. We show an example of this in Fig. 8. By increasing the magnitude and response time of inhibitory feedback (specifically, $G_{i}=-2.0, \alpha_{A}=20 \mathrm{~s}^{-1}, \beta_{A}=5 \mathrm{~s}^{-1}$, with other parameters unchanged, and driving excitatory-to-excitatory axons only) a strong resonance can be introduced that has a peak at $2.5 \mathrm{~Hz}$, as shown in Fig. 8a. Application of TBS has been modelled for 3 pulses per burst, at different frequencies, for the cTBS and iTBS cases. Results are shown in Fig. 8b. This plot shows that the greatest effect occurs when the burst rate is also at $2.5 \mathrm{~Hz}$, the same as the resonant peak in $Q_{e}^{T}$. This suggests that there is some merit in optimizing a TMS protocol to match resonances in a subject's EEG. 

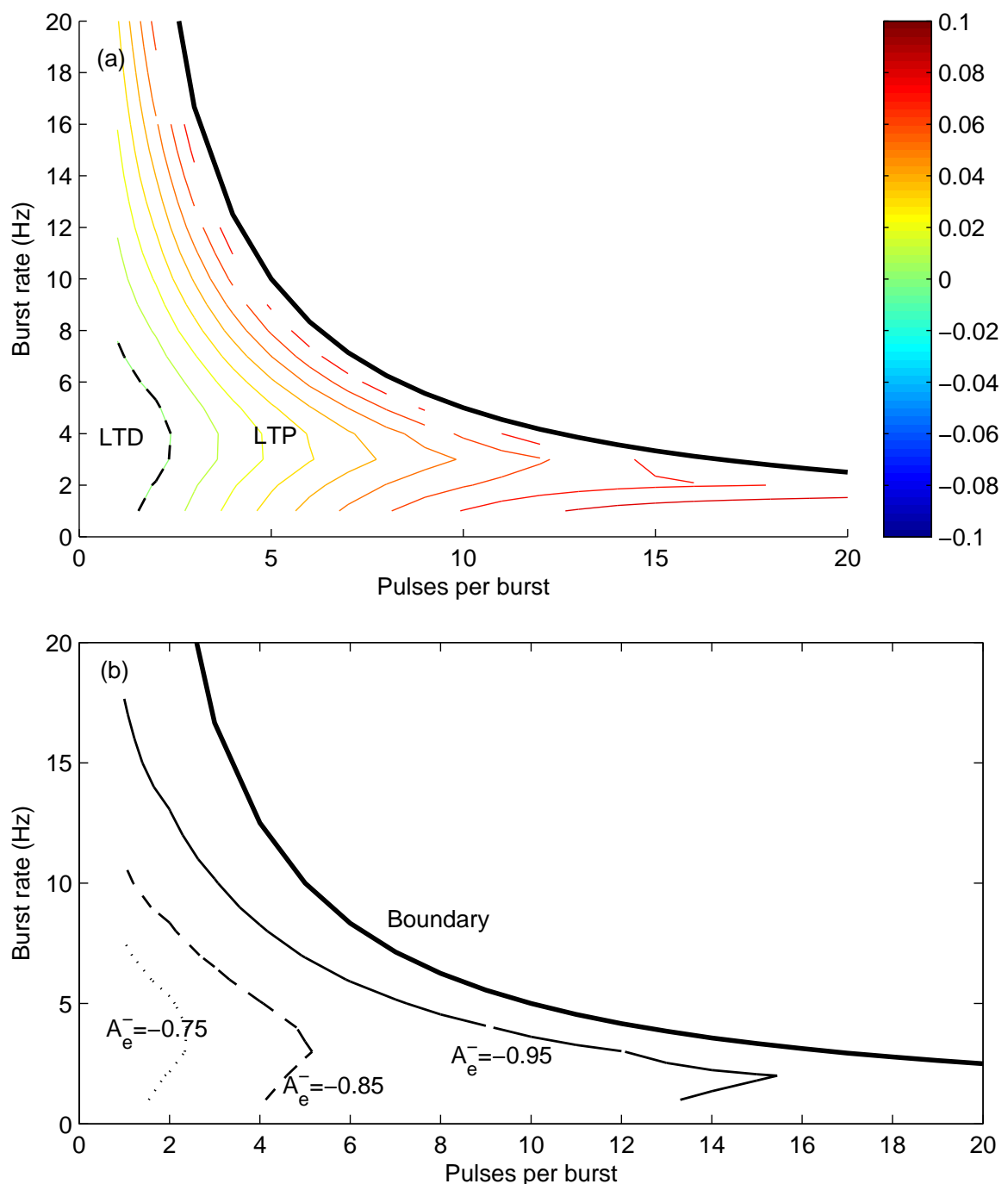

Fig. 5 (a) A plot of $\Delta w_{e e}$ per pulse, from Eq. (24) for intermittent TMS protocols. Results are presented as a contour plot of $\Delta w_{e e}$ per pulse as a function of $50 \mathrm{~Hz}$ pulses per burst (x-axis) against burst rate (y-axis). Key: LTD — Long term depression; LTP — Long term potentiation. (b) A plot of the position of the border between LTD and LTP as a function of $A_{e}^{-}$. The thick solid line shows the limit of the range of possible burst rates and pulses per burst. 


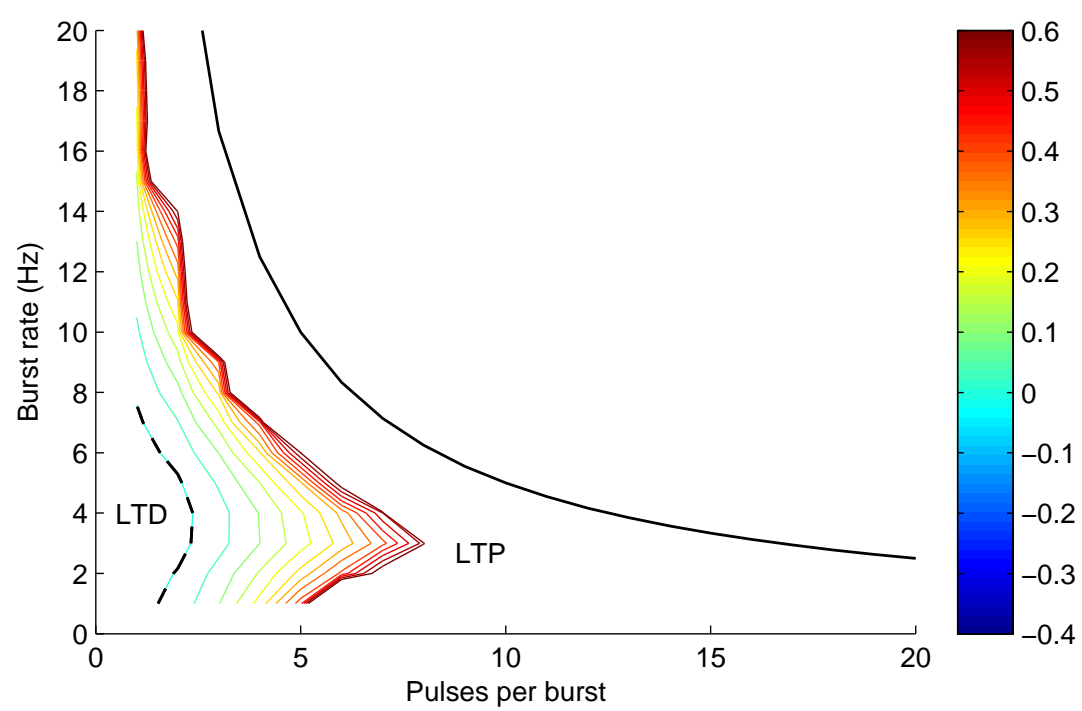

Fig. 6 A plot of the change in $w_{e e}$ over a 600-pulse cTBS protocol as a function of $50 \mathrm{~Hz}$ pulses per burst (x-axis) against burst rate (y-axis). Key: LTD - Long term depression; LTP - Long term potentiation.

We also look at TBS with various time periods in which the TMS is present ('on-epoch') and absent ('off-epoch'). Figure 9 shows the TBS result (3 pulses per burst at $5 \mathrm{~Hz}$ burst rate) for different on and off epochs. If the off epoch is sufficient long, the result does not change for a given on-epoch. However, the degree of potentiation or depression depends significantly on the length of the on-epoch. Shorter on-epochs give a greater degree of potentiation. This is testable experimentally. When on- and off-epochs are of similar length the result is approximately zero (i.e. neither potentiation nor depression) in agreement with the result of Huang et al. [18].

\subsection{Varying the stimulated population}

The model is particularly sensitive to the population of neurons that is driven. Figure 10 shows how the results of the cTBS and iTBS protocols change with a change in driven population. We consider the case of $\lambda_{e e}+\lambda_{i e}=1$ (i.e. the same total axonal stimulation). Part (a) demonstrates the variation in $\left|Q_{e}^{T}(f)\right|^{2}$ for different values of $\lambda_{e e}$. When $\lambda_{e e}$ is small, the response is much lower. Part (b) shows $\Delta w_{e e}$ per pulse as a function of $\lambda_{e e}$ for the iTBS and cTBS protocols of Huang et al. [18]. It is evident that while cTBS usually produces depression and iTBS potentiation, the magnitude of these depend considerably on $\lambda_{e e}$. The effectiveness of both iTBS and cTBS is likely to be low if inhibitory neurons are predominatly driven. Experimentally, Hamada et al. have shown that cTBS and iTBS are most distinct in their effects amongst subjects who have most sensitivity to the orientation of the stimulating TMS coil and suggest that this is attributable to the differences in populations that are stimulated [16]. The model results confirm 

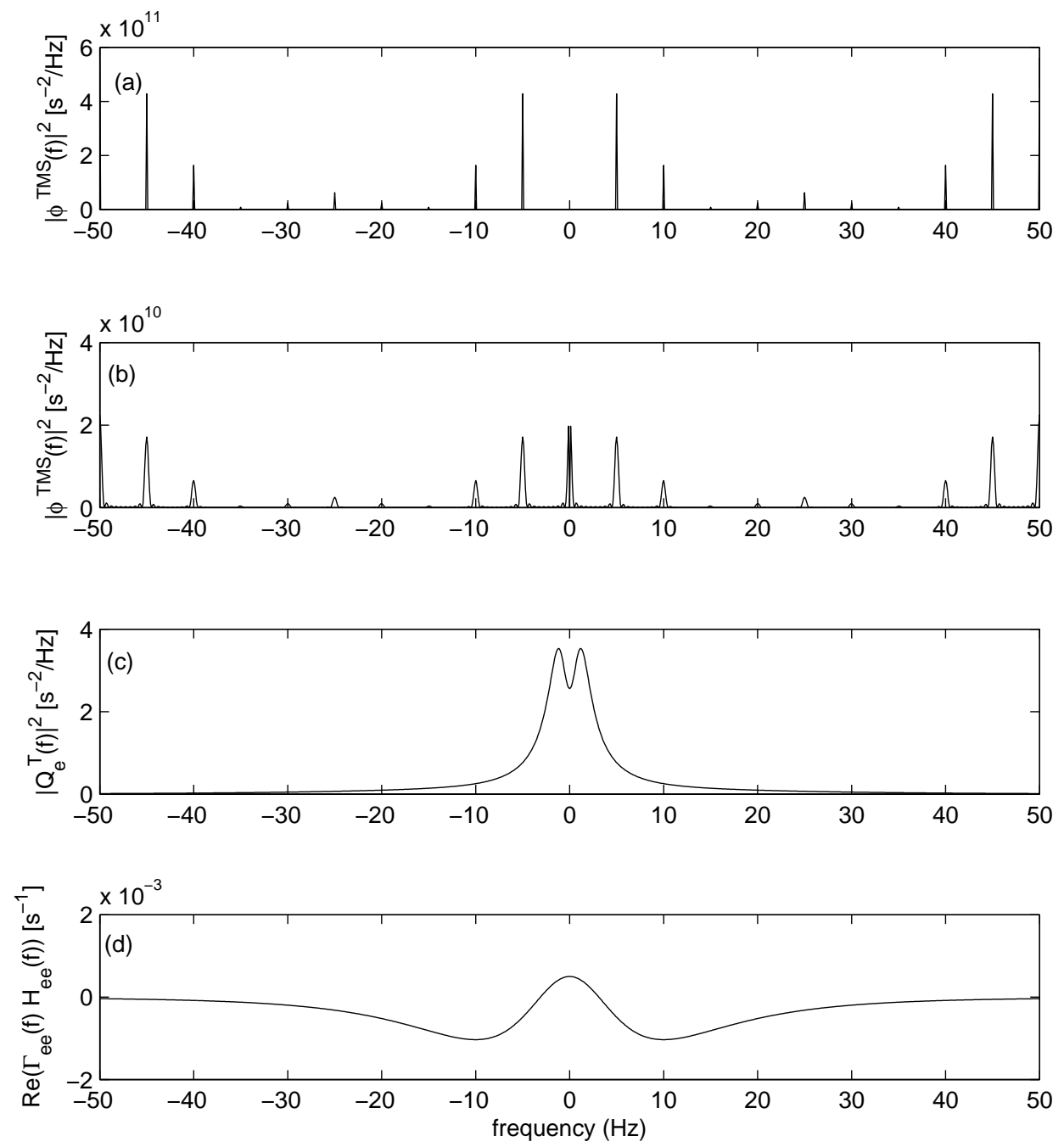

Fig. 7 A plot of the functions that contribute to Eq. (24). (a) The driving function $\left|\phi^{\mathrm{TMS}}(f)\right|^{2}$ against frequency $f=\omega / 2 \pi$ for the cTBS protocol. (b) The driving function $\left|\phi^{\mathrm{TMS}}(f)\right|^{2}$ against frequency for the iTBS protocol. (c) The frequency response of the excitatory cells, $\left|Q_{e}^{T}(f)\right|^{2}$. (d) The plasticity function $\operatorname{Re}\left[\Gamma_{e}(f) H_{e e}(f)\right]$. 
(a)

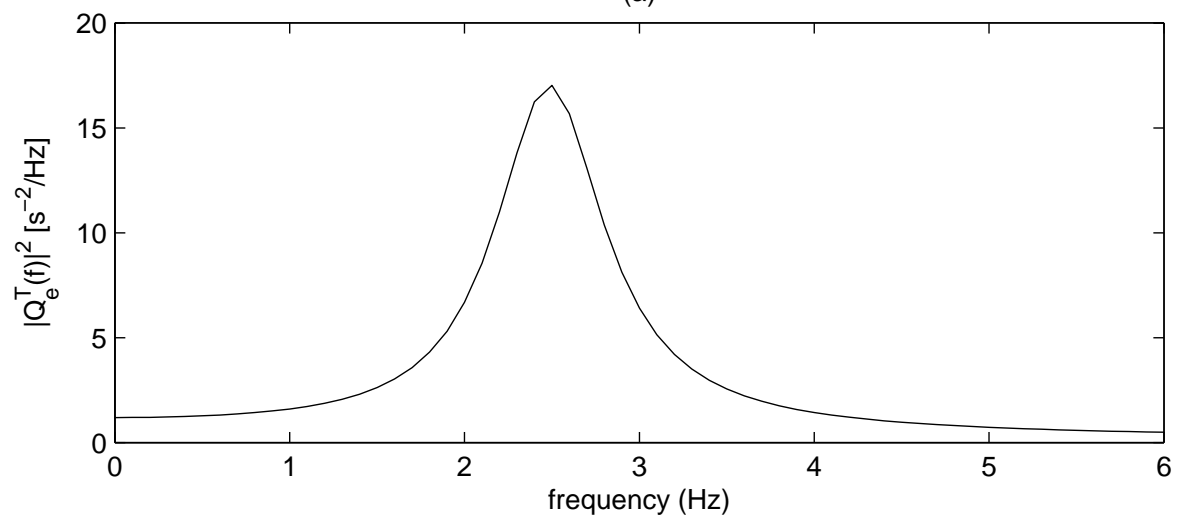

(b)

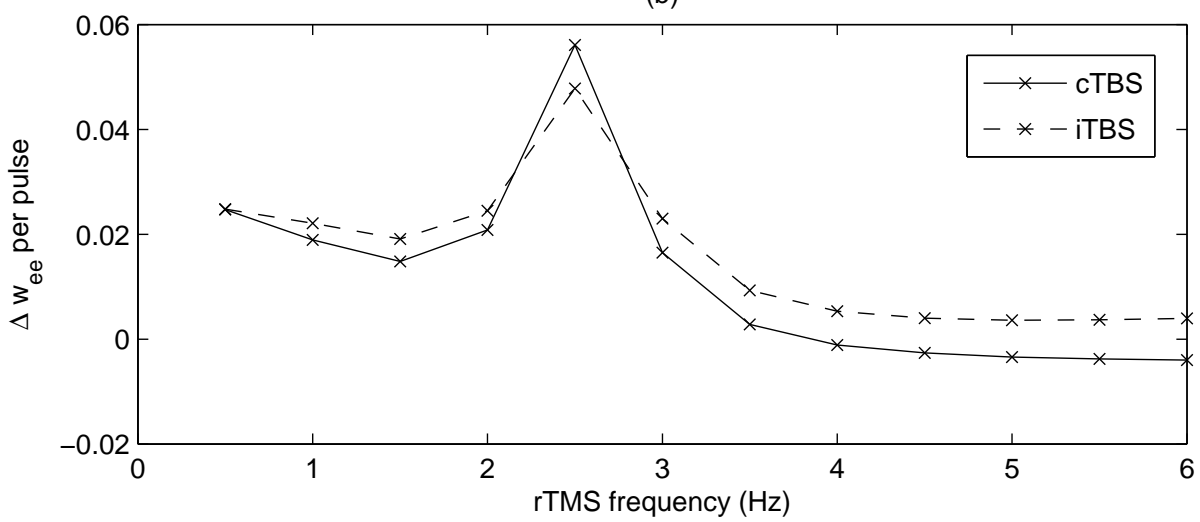

Fig. 8 (a) A plot of the excitatory firing rate response to white noise in the excitatoryto-excitatory axons, $\left|Q_{e}^{T}\right|^{2}$, against frequency. The parameters of Table. 1 have been used, except for $G_{i}=-2.0, \alpha_{A}=20 \mathrm{~s}^{-1}$ and $\beta_{A}=5 \mathrm{~s}^{-1}$. (b) A plot of $\Delta w_{e e}$ per pulse against burst frequency for the cTBS (solid) and iTBS (dashed) protocols, with three pulses at $0.02 \mathrm{~s}$ separation, per burst.

that the stimulated population is an important parameter in determining the effect of TBS.

\subsection{Paired pulse protocol}

Finally we present results for a paired pulse protocol. Figure 11a shows a plot for a continuous train of pulse pairs, repeated every ten seconds. The plot shows $\Delta w_{e e}$ per pulse against the time difference between the pulses in each pair. For time differences less than $15 \mathrm{~ms}$, the plot shows a negative weight change (depression), at intermediate time differences (15-150 ms) there is positive weight change (potentiation), and at larger time differences the change is negative again. The shape 

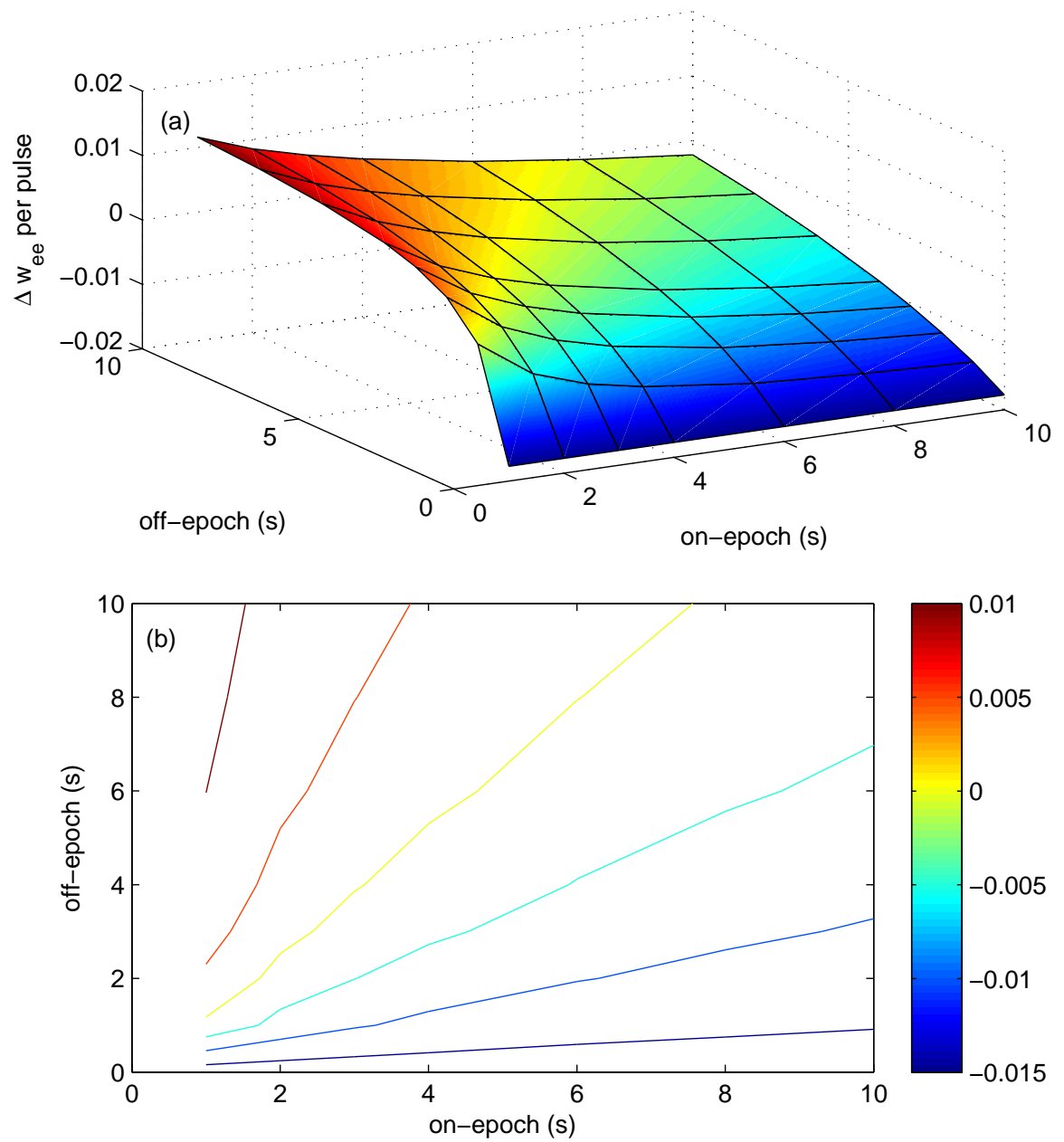

Fig. 9 Surface (a) and contour (b) plots of $\Delta w_{e e}$ per pulse for a TBS protocol of 3 pulses per burst at $5 \mathrm{~Hz}$ burst rate, as a function of on-epoch and off-epoch durations.

of the plot broadly follows what is typically seen in practice with the size of the MEP response. An example of such experimental data collected by the authors from six subjects using the method of Kujirai et al. [22] is shown in Fig. 11b. Intracortical circuits were evaluated using a conditioning intensity of $80 \%$ resting motor threshold and a test intensity that produced MEPs of approximately $50 \%$ maximum amplitude. The effect of interstimulus interval was assessed in a block for each interval, consisting of 10 single (test pulse alone) and 10 paired (conditioning and test pulse) TMS pulses in an intermixed design. Results show that the conditioned MEP response is increased for intervals greater than $5 \mathrm{~ms}$, and returns to baseline at about $40 \mathrm{~ms}$. The results are highly variable from trial to trial, 

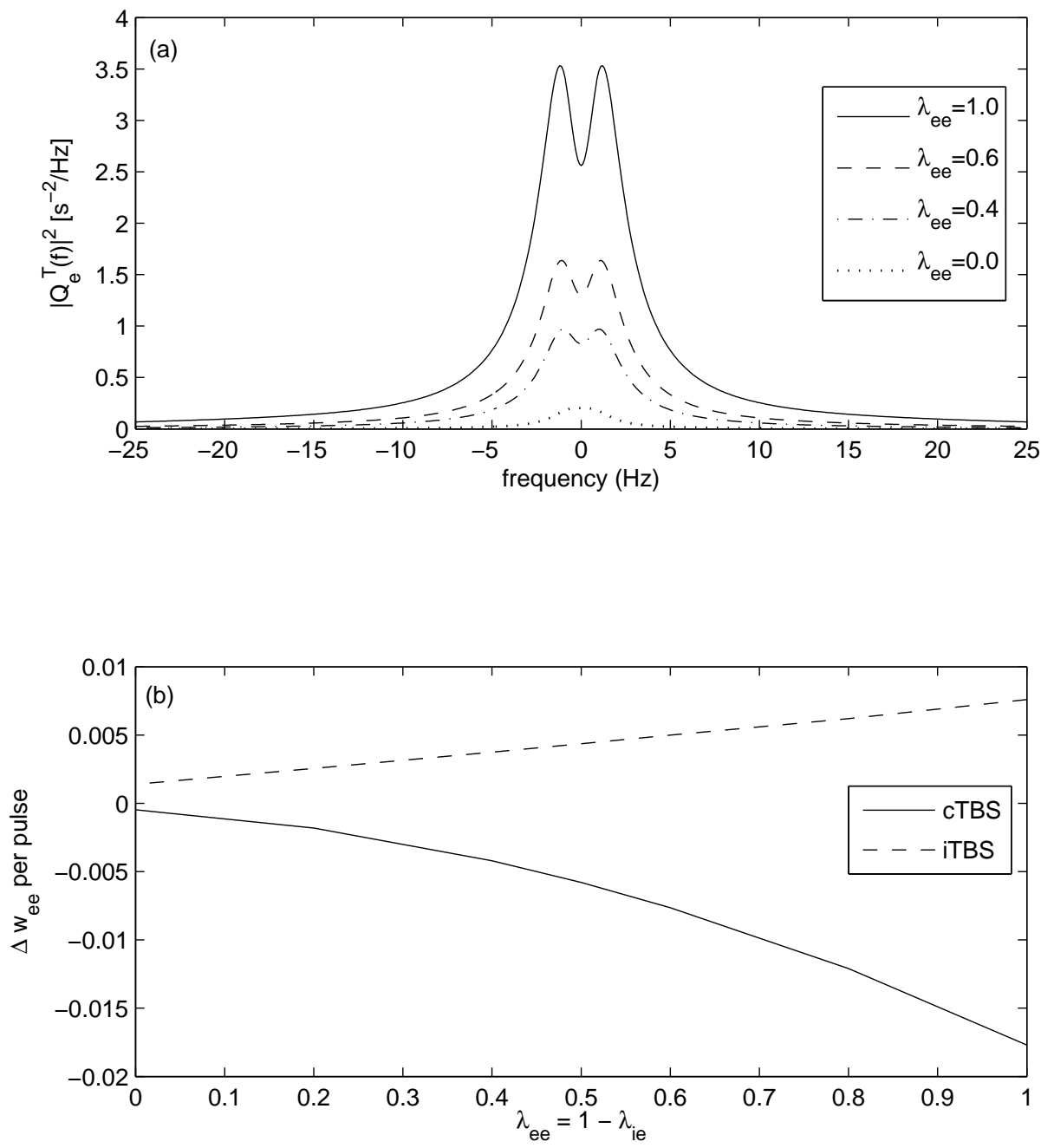

Fig. 10 The effect of varying the population that is driven by the TMS pulses. (a) A plot of the transfer function $\left|Q_{e}^{\mathrm{T}}(f)\right|^{2}$ against $f$ for the cases of $\lambda_{e e}=1.0$ (solid line), $\lambda_{e e}=0.6$ (dashed line), $\lambda_{e e}=0.4$ (dash-dot line) and $\lambda_{e e}=0.0$ (dotted line). In all cases $\lambda_{i e}=1-\lambda_{e e}$ and $\lambda_{e i}=\lambda_{i i}=0$. (b) The result of the cTBS and iTBS protocols of Huang et al. [18] against $\lambda_{e e}$. 
but an ANOVA test shows no statistically significant differences between subjects $(p=0.25)$. The modelled results of (a) are similar in shape to the experiment (b) but timescales are about double what is seen in practice. Additionally, the predicted region of depression at large time intervals is not seen experimentally. A larger conditioning intensity may be needed to show this effect [29]. We emphasize that we have constructed a plot of $\Delta w_{e e}$ against time interval and used this as a proxy for the size of MEP rather than creating a model for the production of the MEP itself. Although the two will be related, we would expect it to be more complicated than a simple proportional scaling.

\section{Conclusion}

We have used a model of STDP within a spatially symmetric neural field theory to model the effects of established TMS protocols on strengths of connections between populations of cortical cells. Although not considered explicitly here, spatial effects such as short wavelength modes of excitation, inter-hemispheric inhibition and a thalamacortical delay loop or more explicit thalamocortical modelling could be included [11]. Similarly, we have assumed stimulation results in events on axons of excitatory cells. However, the model can be generalized to consider stimulation of other axons, or of cells by introducing a $\delta Q_{b}^{\text {ext }}$ term into Eq. (3). We have looked at the effects of the continuous and intermittent TBS protocols of Huang et al. [18], and results broadly correspond with experiment. We show that optimizing a protocol based on an individual's EEG is likely to increase the effectiveness of TMS in changing plasticity. However, given the uncertainty in the mechanisms by which TMS stimulates neuronal populations and the additional uncertainty in the experimental results [16], selecting the form of the model and parameters within the model is difficult and in places speculative. Given the variabilities in experimental determinations, it is important that development of realistic models is taken further, and careful, systematic experimental studies are carried out. Further development of such models in tandem with experimental work should prove a useful and necessary addition for the understanding of TMS.

Acknowledgements We thank Felix Fung and Peter Robinson at The University of Sydney for helpful discussions.

\section{References}

1. Bi, G.Q., Poo, M.M.: Synaptic modifications in clutured hippocampal neurons: dependence on spike timing, synaptic strength, and postsynaptic cell type. Journal of Neuroscience 18, 10,464-10,472 (1998)

2. Bienenstock, E., Lehmann, D.: Regulated criticality in the brain? Advances in complex systems 1, $361-384$ (1998)

3. Bienenstock, E.L., Cooper, L.N., Munro, P.W.: Theory for the development of neuron selectivity: orientation specificity and binocular interation in visual cortex. Journal of Neuroscience 2, $32-48$ (1982)

4. Bojak, I., Liley, D.T.J.: Axonal velocity distributions in neural field equations. PLoS Computational Biology 6, e1000,653 (2010)

5. Chen, R., Classen, J., Gerloff, C., Celnik, P., Wassermann, E.M., Hallett, M., Cohen, L.G.: Depression of motor cortex excitability by low-frequency transcranial magnetic stimulation. Neurology 48, 1398-1403 (1997) 

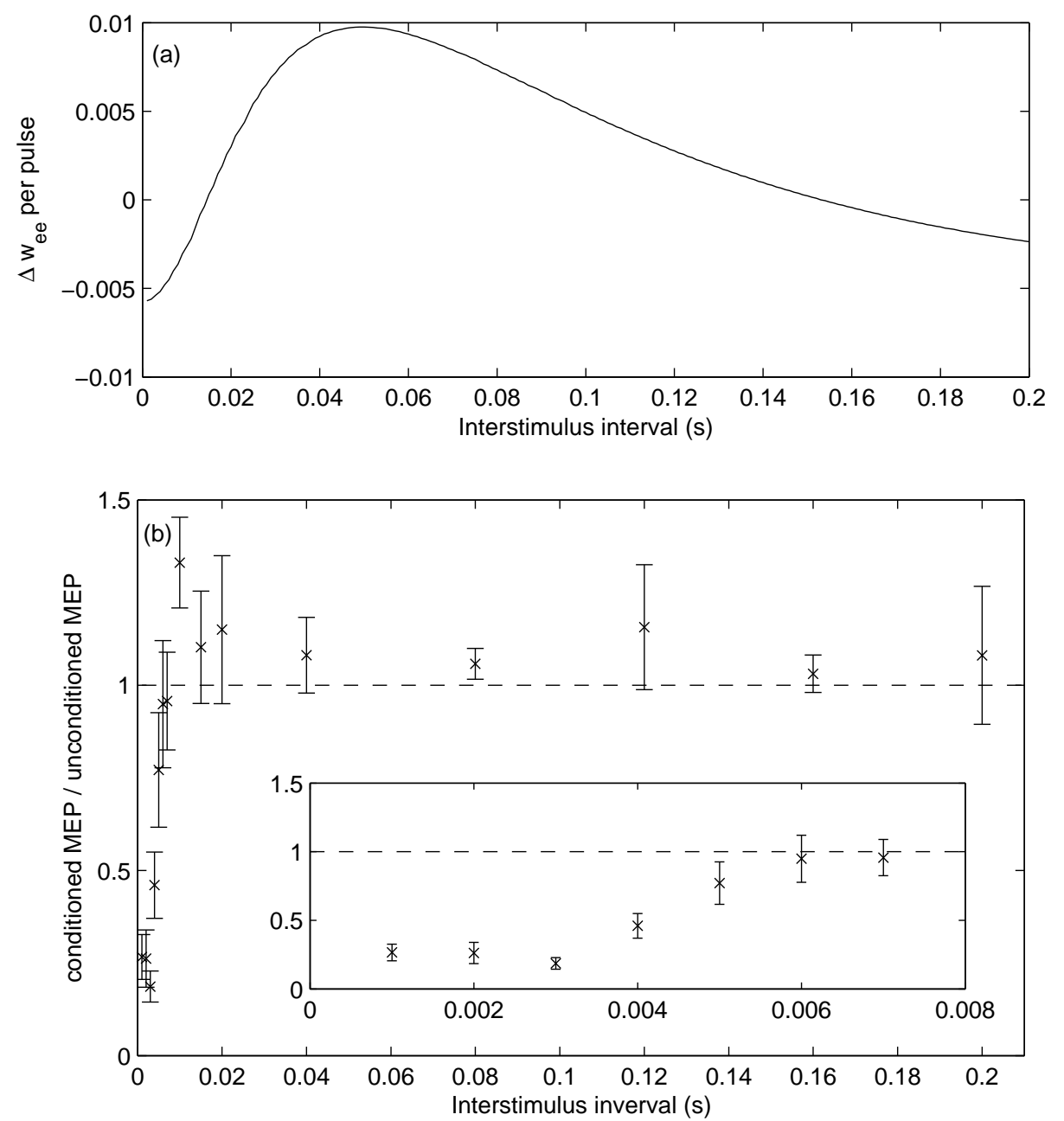

Fig. 11 (a) A plot of $\Delta w_{e e}$ per pulse against time between pulses for a paired pulse protocol. A positive value denotes long-term-potentiation, a negative value long-term-depression. (b) An example of results from a paired-pulse experiment using six subjects with the method of Kujirai et al. [22]. The plot shows the mean MEP size measured at the wrist as a fraction of the MEP produced by the test pulse alone. The error bars show the standard uncertainty in the mean. At small time intervals, the size of the MEP is suppressed (depression), whereas at longer intervals it is increased (potentiation). The insert shows the same data on an expanded scale for small time intervals. 
6. Civardi, C., Collini, A., Monaco, F., Cantello, R.: Applications of transcranial magnetic stimulation in sleep medicine. Sleep Medicine Reviews 13, 35-46 (2009)

7. Deco, G., Jirsa, V.K., Robinson, P.A., Breakspear, M., Friston, K.: The dynamic brain: from spiking neurons to neural masses and cortical fields. Public Library of Science Computational Biology 4(8), e1000,092 (2008)

8. Fitzgerald, P.B., Fountain, S., Daskalakis, Z.J.: A comprehensive review of the effects of rTMS on motor cortex excitability and inhibition. Clinical Neurophysiology 117, 25842596 (2006)

9. Freeman, W.J.: Predictions on neocortical dynamics derived from studies in paleocortex. In: E. Basar, T.H. Bullock (eds.) Induced Rhythms of the Brain, chap. 9, pp. 183-199. Birkhaeuser, Boston (1992)

10. Fregni, F., Simon, D.K., Wu, A., Pascual-Leone, A.: Biophysical mechanisms of multistability in resting-state cortical rhythms. Journal of Neurology, Neurosurgery and Psychiatry 76, 1614-1623 (2005)

11. Fung, P.K., Haber, A.L., Robinson, P.A.: Neural field theory of plasticity in the cerebral cortex. Journal of Theoretical Biology 318, 44-57 (2013)

12. Fung, P.K., Robinson, P.A.: Neural field theory of calcium dependent plasticity with applications to transcranial magnetic stimulation. Journal of Theoretical Biology 324, 72-83 (2013)

13. Gerstner, W., Kempter, R., van Hemmen, J.L., Wagner, H.: A neuronal learning rule for sub-millisecond temporal coding. Nature 386, 76-78 (1996)

14. Gerstner, W., Kistler, W.M.: Spiking neuron models: Single neurons, populations, plasticity. Cambridge University Press (2002)

15. Hallett, M.: Transcranial magnetic stimulation: A primer. Neuron 55, 187-199 (2007)

16. Hamada, M., Murase, N., Hasan, A., Balaratnam, M., Rothwell, J.C.: The role of interneuron networks in driving human motor cortex plasticity. Cerebral Cortex 23, 1593-1605 (2013)

17. Hess, G., Aizenman, C.D., Donoghue, J.P.: Conditions for the induction of long-term potentiation in layer II/III horizontal connections of the rat motor cortex. Journal of Neurophysiology 75, 1765-1778 (1996)

18. Huang, Y.Z., Edwards, M.J., Rounis, E., Bhatia, K.P., Rothwell, J.: Theta burst stimulation of the human motor cortex. Neuron 45, 201-206 (2005)

19. Jirsa, V.K., Haken, H.: A field theory of electromagnetic brain activity. Physical Review Letters 77, 960-963 (1996)

20. Kempter, R., Gerstner, W., van Hemmen, J.L.: Hebbian learning and spiking neurons. Physical Review E 59, 4498-4514 (1999)

21. Koch, C.: Biophysics of Computation: Information Processing in Single Neurons. Oxford University Press, New York (1999)

22. Kujirai, T., Caramia, M.D., Rothwell, J.C., Day, B.L., Thompson, P.D., Ferbert, A., Wroe, S., Asselman, P., Marsden, C.D.: Corticocortical inhibition in human motor cortex. Journal of Physiology 471, 501-519 (1993)

23. Lazzaro, V.D., Oliviero, A., Meglio, M., Cioni, B., Tamburrini, G., Tonali, P., Rothwell, J.C.: Direct demonstration of the effect of lorazepam on the excitability of the human motor cortex. Clinical Neurophysiology 111, 794-799 (2000)

24. Lazzaro, V.D., Pilato, F., Dileone, F., Profice, M., Olivero, A., Mazzone, P., Insola, A., Ranieri, F., Meglio, M., Tonali, P.A., Rothwell, J.C.: The physiological basis of the effects of intermittent theta burst stimulation of the human motor cortex. Journal of Physiology 586, 3871-3879 (2008)

25. Lazzaro, V.D., Pilato, F., Saturno, E., Olivero, A., Dileone, M., Mazzone, P., Insola, A., Tonali, P.A., Ranieri, F., Huang, Y.Z., Rothwell, J.C.: Theta-burst repetitive transcranial magnetic stimulation suppresses specific excitatory circuits in the human motor cortex. Journal of Physiology 565, 945-950 (2005)

26. Liley, D.T.J., Cadusch, P.J., Dafilis, M.P.: A spatially continuous mean field theory of electro-cortical activity. Network 13, 67-113 (2002)

27. Lisman, J., Spruston, N.: Questions about STDP as a general model of synaptic plasticity. Frontiers in Synaptic Neuroscience 2, 140 (2010)

28. Massimini, M., Tononi, G., Huber, R.: Slow waves, synaptic plasticity and information processing: insights from transcranial magnetic stimulation and high-density EEG experiments. European Journal of Neuroscience 29, 1761-1770 (2009)

29. Nakamura, H., Kitagawa, H., Kawaguchi, Y., Tsugi, H.: Intracortical facilitation and inhibition after transcranial magnetic stimulation in conscious humans. Journal of Physiology 498, 817-823 (1997) 
30. Nunez, P.L.: The brain wave function: A model for the EEG. Mathematical Biosciences 21, 279-297 (1974)

31. Oberman, L., Edwards, D., Eldaief, M., Pascual-Leone, A.: Safety of theta burst transcranial magnetic stimulation: A systematic review of the literature. Journal of Clinical Neurophysiology 28, 67-74 (2011)

32. Palmer, L.M., Schulz, J.M., Murphy, S.C., Ledergerber, D., Murayama, M., Larkum, M.E.: The cellular basis of GABA $_{B}$-mediated interhemispheric inhibition. Science 335, 989-993 (2012)

33. Pascual-Leone, A., Valls-Solé, J., Wassermann, E.M., Hallett, M.: Responses to rapid-rate transcranial magnetic stimulation of the human motor cortex. Brain 117, 847-858 (1994)

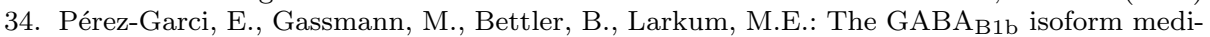
ates long-lasting inhibition of dendritic $\mathrm{Ca}^{2+}$ spikes in layer 5 somatosensory pyramidal neurons. Neuron 50, 603-616 (2006)

35. Ridding, M.C., Rothwell, J.C.: Is there a future for theraputic use of transcranial magnetic stimulation? Nature Neuroscience 8, 559-567 (2007)

36. Robinson, P.A.: Neural field theory of synaptic plasticity. Journal of Theoretical Biology 285, 156-163 (2011)

37. Robinson, P.A., Kim, J.W.: Spike, rate, field and hybrid methods for treating neural dynamics derived from conductance-based equations. Journal of Neuroscience Methods 205, 283-294 (2012)

38. Robinson, P.A., Rennie, C.J., Rowe, D.L., O`Connor, S.: Estimation of multiscale neurophysiologic parameters by electroencephalographic means. Human Brain Mapping 23, 53-72 (2004)

39. Robinson, P.A., Rennie, C.J., Rowe, D.L., O‘Connor, S., Gordon, E.: Multiscale brain modelling. Philisophical Transactions of the Royal Society B 360, 1043 (2005)

40. Robinson, P.A., Rennie, C.J., Wright, J.J.: Propagation and stability of waves of electrical activity in the cerebral cortex. Physical Review E 56, 826-840 (1997)

41. Rothkegel, H., Sommer, M., Paulus, W.: Breaks during $5 \mathrm{~Hz}$ rTMS are essential for facilitatory after effects. Clinical Neurophysiology 121, 426-430 (2010)

42. Rothwell, J.: Techniques of transcranial magnetic stimulation. In: S. Boniface, U. Ziemann (eds.) Plasticity in the human nervous system: Investigations with transcranial magnetic stimulation, pp. 26-61. Cambridge University Press (2003)

43. Shouval, H.Z., Wang, S.S.H., Wittenberg, G.M.: Spike timing dependent plasticity: a consequence of more fundamental learning rules. Frontiers in Computational Neuroscience 4, $19(2010)$

44. Silva, S., Basser, P.J., Miranda, P.C.: Elucidating the mechanisms and loci of neuronal excitation by transcranial magnetic stimulation using a finite element model of a cortical sulcus. Clinical Neurophysiology 119, 2405-2413 (2008)

45. Steyn-Ross, D.A., Steyn-Ross, M.L., Sleigh, J.W., Wilson, M.T., Gillies, I.P., Wright, J.J.: The sleep cycle modelled as a cortical phase transition. Journal of Biophysics 31, 547-569 (2005)

46. Steyn-Ross, M.L., Steyn-Ross, D.A., Wilson, M.T., Sleigh, J.W.: Modeling brain activation patterns for the default and cognitive states. NeuroImage 45, $289-311$ (2009)

47. Talelli, P., Wallace, A., Dileone, M., Hoad, D., Cheeran, B., Oliver, R., VandenBos, M., Hammerbeck, U., Barratt, K., Gillini, C., Musumeci, G., Boudrias, H.H., Cloud, G.C., Ball, J., Marsden, J.F., Ward, N.S., Lazzaro, V.D., Greenwood, R.G., Rothwell, J.C.: Theta burst stimulation in the rehabilitation of the upper limb: A semirandomized, placebocontrolled trial in chronic stroke patients. Neurorehabilitation and Neural Repair 26, 976-987 (2012)

48. Tononi, G., Cirelli, C.: Sleep function and synaptic homeostatis. Sleep Medicine Reviews 10, 49-62 (2006)

49. Touge, T., Gerschlager, W., Brown, P., Rothwell, J.C.: Are the after-effects of lowfrequency rtms on motor cortex excitability due to changes in the efficacy of cortical synapses? Clinical Neurophysiology 112, 2138-2145 (2001)

50. Walsh, V., Cowey, A.: Transcranial magnetic stimulation and cognitive neuroscience. Nature Reviews: Neuroscience 1, 73-79 (2000)

51. Wilson, H.R., Cowan, J.D.: Excitatory and inhibitory interactions in localized populations of model neurons. Biophysical Journal 12, 1-24 (1972)

52. Wilson, M.T., Robinson, P.A., O'Neill, B., Steyn-Ross, D.A.: Complementarity of spikeand rate-based dynamics of neural systems. PLoS Computational Biology 8, e1002,560 (2012) 
53. Wilson, M.T., Steyn-Ross, D.A., Sleigh, J.W., Steyn-Ross, M.L., Wilcocks, L.C., Gillies, I.P.: The k-complex and slow oscillation in terms of a mean-field cortical model. Journal of Computational Neuroscience 21, 243-257 (2006)

54. Ziemann, U., Rothwell, J.C., Ridding, M.C.: Interaction between intracortical inhibition and facilitation in human motor cortex. Journal of Physiology 496, 873-881 (1996) 